EFEITOS DA DENSIDADE DE PLANTIO SOBRE A QUALIDADE DE CRAVOS, Dianthus caryophyzzus L. *

MARIA ALICE DE LOURDES BUENO SOUSA **

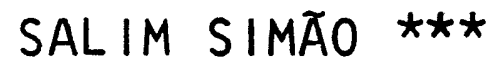

RESUMO

O trabalho teve como objetivo estudar a influência de diferentes densidades populacio nais de craveiros, cultivados em casa de vé getação sobre a qualidade das flores.

As densidades de plantio estudadas foram de $233.333,175.000$ e 116.667 plantas por hectare, obtidas da utilização dos seguintes espaçamentos: $0,15 \mathrm{~m} \times 0,20 \mathrm{~m} ; 0,20 \mathrm{~m} \times 0,20 \mathrm{~m}$ e $0,30 \mathrm{~m} \times 0,30 \mathrm{~m}$. Scania Red foi o cultivar utilizado. Os parâmetros analisados foram o nümero de cravos com cálice inteiro e rachado por planta e por hectare, bem como comprimento da haste com flor, peso da haste com flor e diâmetro da corola.

* Entregue para publicação em 09/12/1981.

Parte da tese de mestrado apresentada pelo primeiro autor à E.S.A. "Luiz de Queiroz", USP.

** Departamento de Horticultura, F.C.A., UNESP, Campus de Botucatu.

$\star \star \star$ Departamento de Agricultura e Horticultura, E.S.A. "Luiz de Queiroz"', USP. 
Concluiu-se que o aumento da densidade de plantio ocasionou um acréscimo na produção de cravos com cálice inteiro por hectare, uma diminuição da produção de cravos com cálice rachado por planta, uma diminuição do peso da haste com flor e uma diminuição do diâmetro da corola. Não houve influência da densidade de plantio sobre a produção de cravos com cálice inteiro por planta, sobre a produção de cravos com cálice rachado por hectare e sobre o comprimento da haste com flor.

INTRODUÇÃO

o craveiro Dianthus caryophyZZus L., espécie da familia Caryophyllaceae, é uma das mais importantes floriferas de cor te. Originário de uma extensa região que abrange desde o sui da Europa até a India, encontrou em regiōes de clima mais ame no do Brasil, condições favoráveis para seu cultivo.

Apesar de sua importância econômica, poucos estudos exị tem no pais, sobre a cultura. Em outros países, pesquisas sobre o cultivo de cravos são feitas mais intensamente. Entretanto, a extrapolação dessas experiências para as nossas culturas nem sempre é viāvel, devido às condições climáticas diferentes.

Neste trabalho, estudou-se a influência de diferentes densidades de plantio sobre a qualidade das flores de craveiros.

\section{REVISÃO DA LITERATURA}

A densidade de plantio é importante fator de produção. A variação desse fator causa modificações no comportamento da população, no häbi to das plantas e, consequentemente, na produção. 
Assim, os diferentes órgãos da planta podem reagir a pressões de populações crescentes, após iniciada a competição, pela diminuição do seu tamanho, nümero ou ambos, afetando não só a produtividade como a qualidade do produto (JANICK, 1.966).

LOCKIE \& BUTTERS (1956) não encontraram diferenças significativas na qualidade das flores produzidas nos espaçamentos por eles testados em três cultivares de craveiros. Resultados semelhantes foram obtidos por HOLLEY \& LEHMAN (1961) e LOCKIE (1961). KIPLINGER (1963) evidenciou apenas pequenas di ferenças na qualidade das flores produzidas no experimento em que foram comparados o sistema convencional de plantio de craveiros $(0,20 \mathrm{~m} \times 0,23 \mathrm{~m})$ e o sistema Weirich.

KOON (1962) relatou que a qualidade dos cravos è obtida às expensas da produção pelo decréscimo da densidade de plantio. Esse mesmo fato foi confirmado por SEAGER (1965) e GARCIA \& AZURMENDI (1971). KOON (1962) ressaltou ainda que o sis tema Weirich de espaçamento è o que melhor confere qualidadé à flor durante o inverno devido a maior iluminação entre as plantas. SHEARD \& BUNT (1963) e SHEARD (1967) obtiveram uma redução geral na qualidade das flores nos plantios de setembro, janeiro e março.

DURKIN \& JANICK (1966), testando diferentes densidades de plantio de craveiros, não encontraram diferenças significa tivas na qualidade das flores produzidas no primeiro pico dé produção, setembro, outubro e novembro, mas a qualidade decli nou linearmente com o aumento da densidade para o segundo é terceiro picos de produção, respectivamente, dezembro, janeiro e fevereiro e março, abril e maio. O efeito da densidade de plantio na qualidade das flores produzidas, de acordo com a época da cultura, foi também constatada por BUNT \& SHEARD (1967), concluindo que alta densidade afeta adversamente a qualidade da flor no plantio de julho mas não apresenta efeito significativo sobre a qualidade em outras épocas. A propor ção de flores do primeiro tipo de qualidade em relação a flo= res do segundo tipo variou de 1,8 para o plantio de julho a 5, 4 para o de setembro. Esses autores atribuiram as diferenças periódicas na qualidade das flores ao fotoperiodo, à intensidade de luz e à idade das plantas. Segundo VONK-NOORDE- 
GRAAFF (1969), a qualidade do cravo è bastante reduzida quando se utiliza uma densidade maior que 168.000 plantas por hectare.

Com respeito à qualidade da haste floral, KOON encontrou melhor qualidade em densidades menores durante o periodo de inverno.

SEAGER (1969) não encontrou efeito do espaçamento sobre o comprimento da haste floral e o peso de cravos de plantas despontadas do cultivar White Sim, mas a qualidade das flores terminais desse mesmo cultivar melhorou quando houve aumento no espaçamento.

Os relatos da literatura a respeito da influência do es paçamento na proporção produzida dos diferentes tipos de qualidade de cravos são conflitantes.

WELKE PLANTHOEVEELHEDEN (1968), em experimento com värias densidades de plantio, observou menor produção de flores do primeiro tipo de qualidade nas densidades de $240.000, \ldots$ 260.00 e 640.000 plantas por hectare em relação à maior produção desse mesmo tipo na densidade de 480.000 plantas por hec tare. Por outro lado, altas densidades de plantio influenciaram levemente a produção de flores do primeiro tipo de qualidade e aumentaram muito a produção de flores do segundo e ter ceiro tipos em experimentos de KINNINGS (1965) e FAIRFIELD (1965).

SHEARD \& BUNT (1963), SHEARD (1967), SEAGER (1969) e JENSEN (1973) não detectaram diferenças na produção de flores de primeiro tipo de qualidade na dependencia das densidades de plantio testadas. Esses autores, porém, evidenciaram uma maior produção de flores do segundo e terceiro tipos em altas densidades de plantio.

MENHENETT et alii (1967) combinaram diferentes densidades de plantio com diferentes severidades de corte de flores. 0 número de flores do primeiro tipo de qualidade não variou com os tratamentos. 0 aumento na produção total em altas densidades de plantio foi principalmente devido ao maior número de flores do segundo tipo de qual idade produzidas. 
Um dos problemas que afetam a qualidade do cravo è a rachadura do cálice. Se a rachadura ocorrer em dois ou mais lugares, a fenda é curta e a flor apresenta valor comercial. Se a rachadura ocorre em um só lado, ela è geralmente grande (maior que a metade do comprimento do cálice). Nesse caso, as sépalas adjacentes se separam e as pétalas se curvam para bai xo, destruindo a forma e a arquitetura da flor, resultando ná perda do seu valor comercial. Dependendo do número de pétalas o cravo pode ser simpiles - 5 pétalas, dobrado -30-60 pétalas ou super dobrado - 100 a 350 pétalas (Batchelor, citado por SZENDEL, 1937). A rachadura do cālice é característica de cra vos super dobrados, visto estar relacionada ao número de pétalas. Comercialmente, a maioria dos cultivares de craveiros são hibridos entre tipos simples e super dobrados, apresentan do f'lores dobradas, o que tende a diminuir a incidencia de rā chadura em relação ao tipo super dobrado (SZENDEL, 1937). Apé sar da tendência de rachamento ser hereditária, fatores ambientais podem alterar o número de pétalas afetando, assim, a incidência de rachadura do cálice (CONNORS, 1916). Alguns des ses fatores são altas quantidades de fertilizantes, excessō de àgua e flutuações de temperatura (CONNORS, 1916; LAURIE $\varepsilon$ RIES, 1950).

Com respeito à influência da densidade na incidencia de rachadura, FAIRFIELD (1965) obteve uma proporção de $1: 7,5$ de cravos rachados para não rachados em plantios densos. Essa pro porção aumentou para 1:4,7 em culturas menos densas.

Trabalho de SEAGER (1969) demonstrou que a porcentagem média de rachadura em nove cultivares de craveiros, que incluia White Sim, plantados em julho aumentou de $12 \%$ para $16 \%$ quando a densidade aumentou de 241.546 para 427.350 plantas por hectare. Contudo, na densidade de plantio de 694.444 plan tas por hectare o aumento da porcentagem de cálice rachado fó $\bar{i}$ de $23 \%$ em relação à menor densidade. A tendência de rachadura não se repetiu em plantio de setembro, quando a porcentagem de rachadura foi baixa.

MATERIAL E METODOS

o trabalho foi conduzido em casa de vegetação com cobertura de polietileno na Fazenda Experimental São Ma- 
nuel, pertencente à Faculdade de Ciências Agronômicas, "Cam pus" de Botucatu da UNESP.

O substrato usado nos canteiros, constou de solo superficial retirado de mata. A esse solo foi adicionado esterco de curral e calcário dolomítico na base de 35 t/ha e 2 t/ha, respectivamente. Esse substrato foi tratado com brometo de metilo e dibromocloropropano, afim de desinfetar o solo de fun gos e bactérias causadores de doenças. A adubação foi aplicada parceladamente, durante todo o ciclo da cultura, na base de $310 \mathrm{~kg}$ de $\mathrm{N}, 625 \mathrm{~kg}$ de $\mathrm{P}_{2} \mathrm{O}_{5}$ e $70 \mathrm{~kg}$ de $\mathrm{K}_{2} \mathrm{O}$ por hectare.

Scania Red Sim foi o cultivar utilizado, cujas flores vermelhas, apresentam maior aceitação no mercado. 0 plantio efetuou-se em $11 / 01 / 72$, utilizando-se mudas uniformes obtidas do enraizamento prévio de brotações laterais.

Vinte dias após o plantio foi iniciado o desponte nas plantas com seis internódios e posteriormente procedeu-se ao desbaste do número de ramificações laterais, deixando-se as cinco melhores brotações por planta. Eliminou-se, também, os botões florais secundários que surgiram junto ao botão princí pal.

Utilizou-se de um delineamento experimental de blocos casualizados, com sete repetições, com teste "F" e teste Tukey a 5\% de probabilidade (PIMENTEL GOMES, 1976).

As densidades de plantio estudadas foram: $233.333 ; \ldots$ 175.000 e 116.667 plantas por hectare, obtidas pela utiliza ção dos espaçamentos: $0,15 \mathrm{~m} \times 0,20 \mathrm{~m} ; 0,20 \mathrm{~m} \times 0,20 \mathrm{~m} \quad \mathrm{e}$ $0,30 \mathrm{~m} \times 0,20 \mathrm{~m}$, respectivamente. Esse número de plantas por hectare representa o número possível de plantas, cultivadas em um hectare, supondo a perda de $30 \%$ para a área de serviços.

Os dados coletados no experimento, referem-se ao primei ro período de florescimento, à produção de flores obtida noś ramos laterais primários desenvolvidos após o desponte. As flores eram colhidas abertas, com a haste floral com seis internódios, a partir da base do cálice.

Do número total de flores de cada colheita foram separa 
das e contadas as com cálice inteiro e rachado. Nas flores com cálice inteiro avaliou-se o comprimento da haste com flor, o peso da haste com flor e o diâmetro da corola do cravo.

\section{RESULTADOS}

\section{Produção de cravos com cálice inteiro}

0 teste $F$ foi significativo com as produções de cravos com cálice inteiro por hectare, por densidade de plantio.

As médias de produção de cravos com cálice inteiro por hectare, na dependência da densidade utilizada, encontram - se na Tabela 1.

Tabela 1 - Médias da produção total de cravos com cálice inteiro por hectare, expressas pelo número de flo res por hectare, de acordo com a densidade de plan tio.

\begin{tabular}{cc}
\hline $\begin{array}{c}\text { Densidade }(D) \\
\text { (no de plantas/ha) }\end{array}$ & $\begin{array}{c}\text { Nümeros de cravos } \\
\text { com călice inteiro/ha }\end{array}$ \\
\hline 233.333 & $835.415 \mathrm{a}$ \\
175.000 & $641.667 \mathrm{~b}$ \\
116.667 & $409.376 \quad \mathrm{c}$ \\
\hline
\end{tabular}
$\Delta(D)=44.710$ Médias seguidas de letras iguais não diferem entre si pelo teste de Tukey, ao nivel de $5 \%$ de probabilidade.

As médias de produção de cravos com cālice inteiro nas diferentes densidades diferiram entre si pelo teste de Tukey. A produção de flores inteiras aumentou com o aumento da densidade de plantio. 
A produção de cravos com cālice inteiro por hectare, em função dos espaçamentos estudados, è expressa pela equação:

$Y=1.727 .074,44-7.496 .459,70 X+10.347 .106,00 X^{2}$

onde $Y=$ número de cravos com cálice inteiro por hectare $X=$ espaçamentos (Figura 1)

$\mathrm{Na}$ Tabela 2, encontram-se as médias de produção de cravos com cālice inteiro por planta. Os valores de $F$ não foram significativos ao nivel de $5 \%$ de probabilidade para essa caracteristica estudada. Isso significa que, para a produção de cravos com cálice inteiro por planta, não foi constatada influência das densidades de plantio estudadas.

Tabela 2 - Médias da produção de cravos com cálice inteiro por planta, de acordo com a densidade de plantio.

\begin{tabular}{cc}
\hline $\begin{array}{c}\text { Densidade (D) } \\
\text { (no de planta/ha) }\end{array}$ & $\begin{array}{c}\text { Número médio de cravos } \\
\text { com călice inteiro }\end{array}$ \\
\hline 233.333 & 3,58 \\
175.000 & 3,67 \\
116.667 & 3,54 \\
\hline
\end{tabular}

$\Delta(D)=0,26$, ao nivel de $5 \%$ de probabilidade.

Produção de cravos com cálice rachado

Na Tabela 3, observaram-se as médias de produção de cra vos com cálice rachado por hectare. Não foram observadas di= ferenças significativas para as variáveis experimentadas para essa característica.

$\mathrm{Na}$ Tabela 4, apresentam-se as médias referentes à produ ção de cravos com cálice rachado por planta, de acordo com as densidades de plantio experimentadas. 


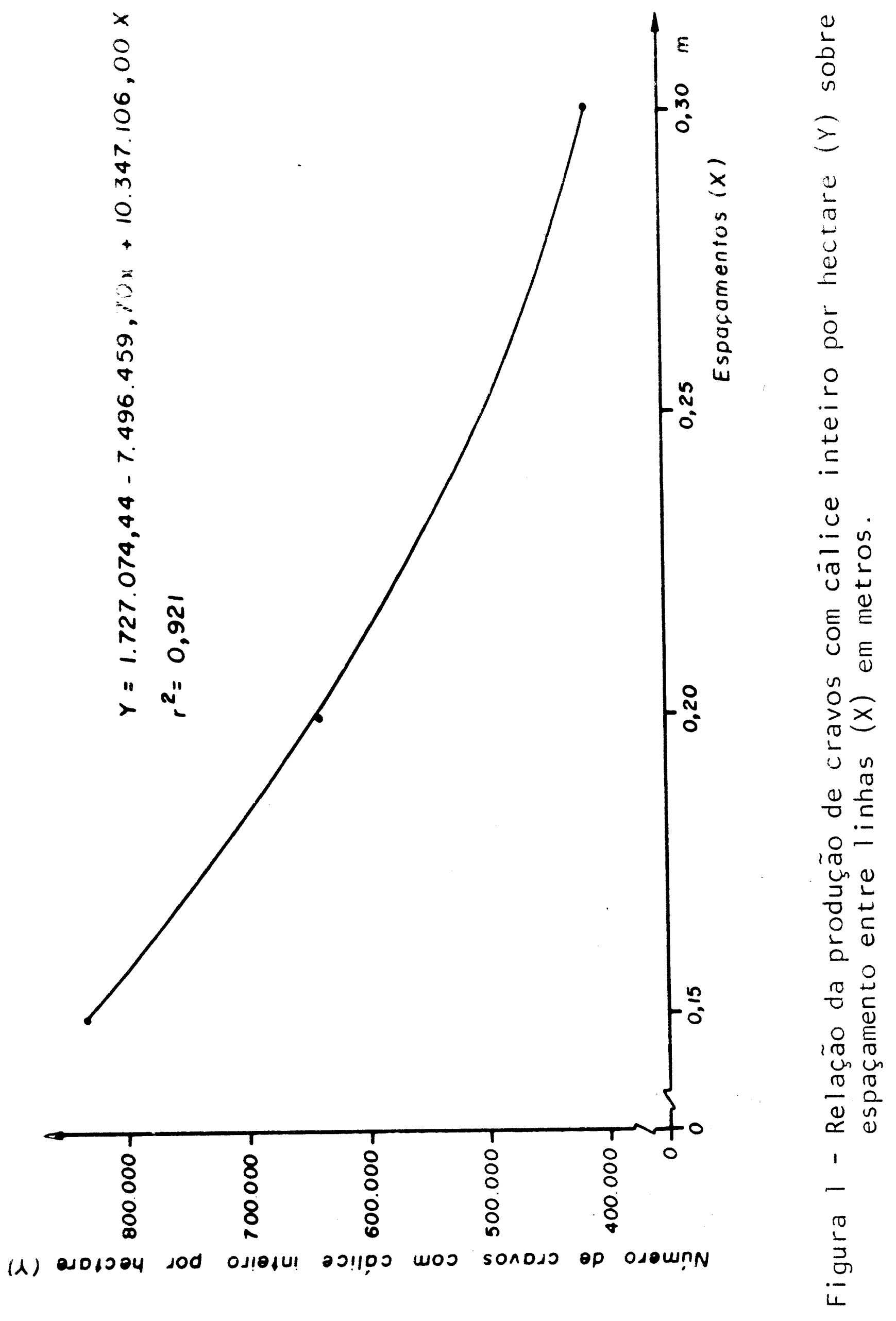


Tabela 3 - Médias da produção de cravos rachados por hectare, de acordo com a densidade de plantio.

\begin{tabular}{cc}
\hline $\begin{array}{c}\text { Densidade }(\mathrm{D}) \\
(\mathrm{n} \text {. de plantas/ha) }\end{array}$ & $\begin{array}{c}\text { Nümero de cravos rachados } \\
\text { por hectare }\end{array}$ \\
\hline 233.333 & 177.951 \\
175.000 & 158.854 \\
116.667 & 138.310 \\
\hline
\end{tabular}

$\Delta(D)=42.174$ ao nivel de $5 \%$ de probabilidade.

Tabela 4 - Médias da produção de cravos com cālice rachado por planta, de acordo com a densidade de plantio.

\begin{tabular}{|c|c|}
\hline $\begin{array}{c}\text { Densidade ( } D) \\
\text { (n: de plantas/ha) }\end{array}$ & $\begin{array}{l}\text { Número médio de cravos } \\
\text { com cálice rachado }\end{array}$ \\
\hline $\begin{array}{l}233.333 \\
175.000 \\
116.667\end{array}$ & $\begin{array}{l}0,76 \text { a } \\
0,91 \text { a } \\
1,18 \text { b }\end{array}$ \\
\hline
\end{tabular}

$\Delta(D)=0,27$ Médias seguidas de letras iguais não diferem entre si pelo teste de Tukey, ao nivel de $5 \%$ de probabilidade.

O teste $F$ mostrou diferenças significativas na produção de cravos com cálice rachado por planta para as densidades de plantio experimentadas.

As médias de produção de cravos com cālices rachados por plantas nas diferentes densidades diferiram entre si pelo tes te Tukey a 5\%. A produção de cravos com cálices rachados pór planta é inversamente proporcional à densidade de plantio. A 
maior densidade (233.333 plantas por ha) e a densidade de 175.000 plantas por hectare não apresentaram diferenças nas médias de produção de flores por planta.

A produção de cravos com cálice rachado por planta em função dos espaçamentos estudados é expressa pela equação: $Y=0,3442+2,8082 X$, onde $Y=$ número de cravos com cálice rachado por planta e $X=$ espaçamentos (Figura 2 ).

Parâmetros de qualidade de cravos com cálice inteiro

Os valores do comprimento médio da haste com flor, do peso médio da haste com flor e do diâmetro médio da corola en contram-se respectivamente nas Tabelas 5,6 e 7 .

Tabela 5 - Comprimentos médios das hastes de cravos com cälice inteiro, de acordo com a densidade de plantio.

\begin{tabular}{cc}
\hline Densidade (D) & Comprimento \\
(no de planta/ha) & media (m) \\
\hline 233.333 & 0,57 \\
175.000 & 0,58 \\
116.667 & 0,57 \\
\hline
\end{tabular}

$\Delta(D)=1,20$, ao nivel de $5 \%$ de probabilidade

0 teste $F$ detectou diferenças significativas ao nivel de $5 \%$ de probabilidade entre as densidades para peso de haste com flor e diâmetro da corola. Não foi constatada diferença significativa entre as densidades para comprimento da haste.

0s pesos médios das hastes com flor para as densidades de 233.333 e 175.000 plantas por hectare não diferiram entre si mas foram significativamente diferentes do peso médio ob- 


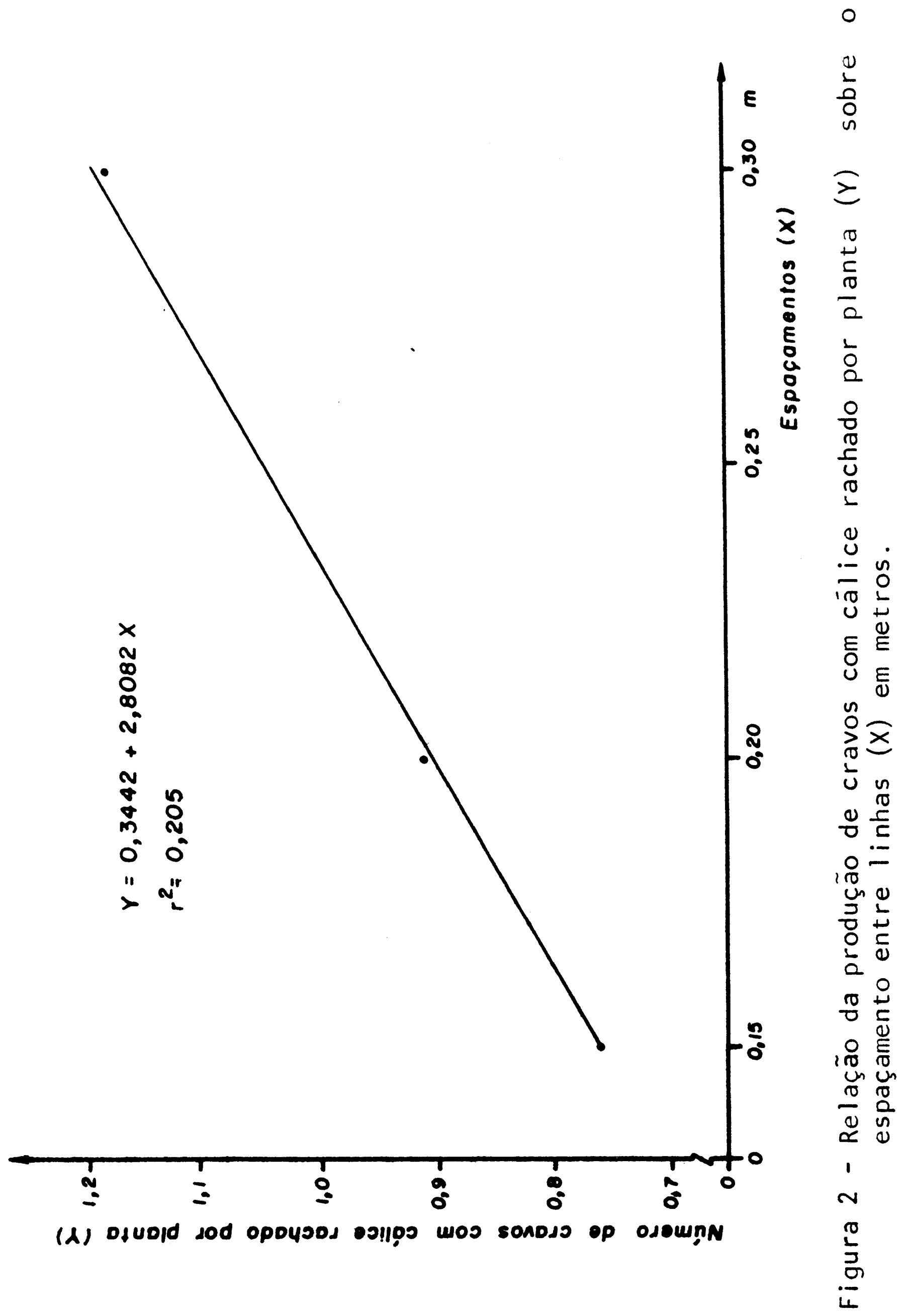


Tabela 6 - Pesos médios das hastes de cravos com cálice inte! ro, de acordo com a densidade de plantio.

\begin{tabular}{cc}
\hline $\begin{array}{c}\text { Densidade (D) } \\
\text { (no de plantas/ha) }\end{array}$ & Peso médio \\
\hline 233.333 & $19,47 \mathrm{a}$ \\
175.000 & $20,01 \mathrm{a}$ \\
116.667 & $20,73 \mathrm{~b}$ \\
\hline
\end{tabular}

$\Delta(D)=0,64$ Mëdias seguidas de letras iguais não diferem entre si pelo teste de Tukey, ao nível de $5 \%$ de probabilidade

Tabela 7 - Diâmetros médios das corolas de cravos com cálice inteiro, de acordo com a densidade de plantio.

\begin{tabular}{cc}
\hline $\begin{array}{c}\text { Densidade }(D) \\
\text { (n: de plantas/ha) }\end{array}$ & $\begin{array}{c}\text { Diâmetro médio } \\
(\mathrm{cm})\end{array}$ \\
\hline 233.333 & $6,17 \mathrm{a}$ \\
175.000 & $6,24 \mathrm{ab}$ \\
116.667 & $6,28 \mathrm{~b}$ \\
\hline
\end{tabular}

$\Delta(D)=0,07$ Médias seguidas de letras iguais não diferem entre si pelo teste de Tukey, ao nivel de $5 \%$ de pro babilidade.

tido na densidade de 116.667 plantas por hectare de acordo com o teste de Tukey, a 5\%.

0s diâmetros médios das corolas, nas densidades de 233.333 e 116.667 plantas por hectare forari significativamente diferentes pelo teste de Tukey a $5 \%$.

A relação entre o peso da haste com flor e os espaçamen tos é expressa pela equação: $Y=18,29+8,23 X$ onde $Y=$ pesó da haste com flor e $X=$ espaçamentos (Figura 3 ). 


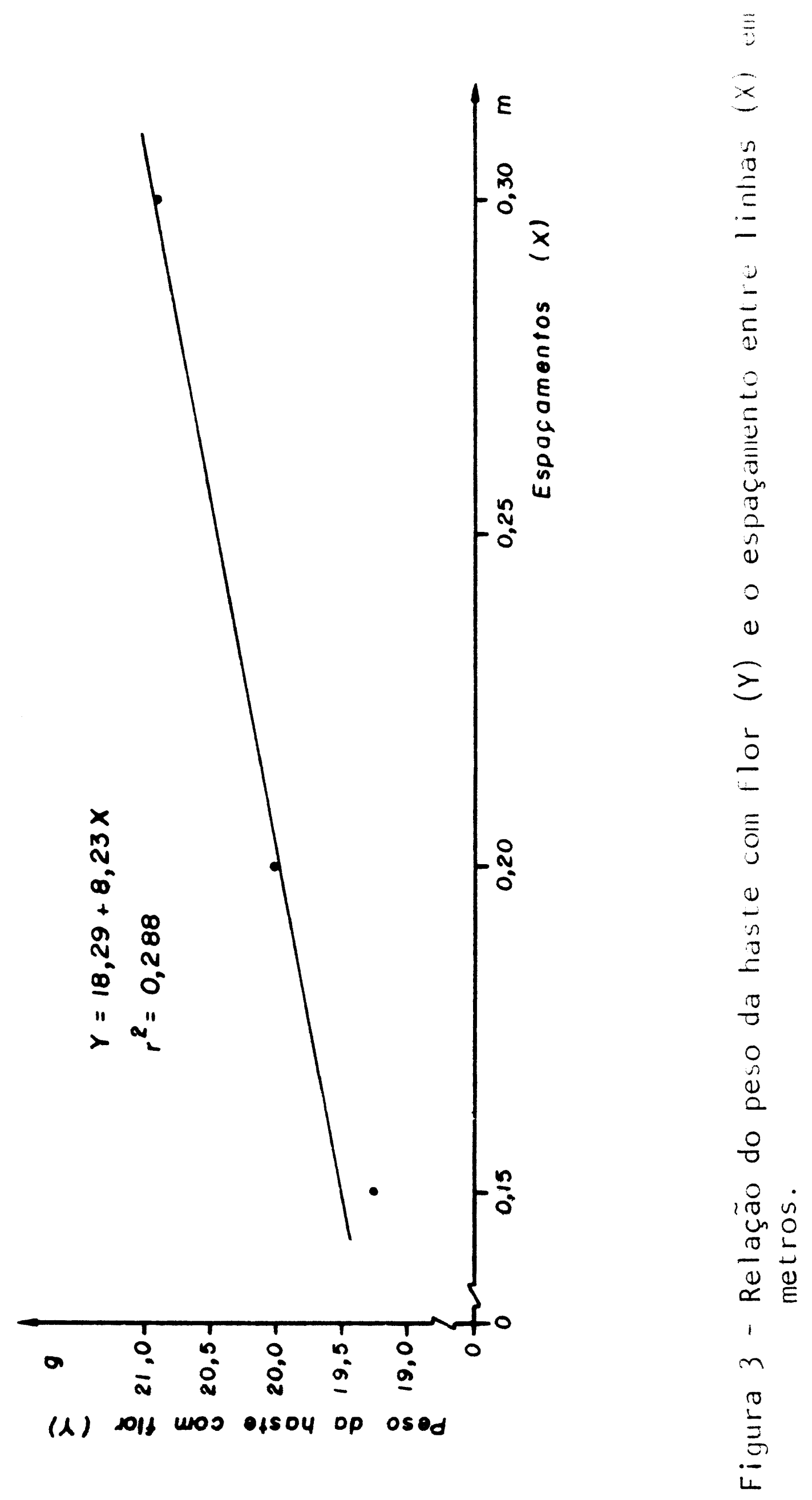




\section{DISCUSSÃO}

Analisando a produção de cravos inteiros por planta (Ta bela 2), nota-se que não houve diferenças entre as densidadeśs estudadas, contudo as produções de cravos inteiros por hectare foram diferentes entre si e aumentaram com a densidade de plantio (Tabela 1 e Figura 1). Esse fato pode ser expiicado, porque pequenas diferenças na produção individual das plantas são grandemente aumentadas quando se considera o acréscimo no nümero de plantas por unidade de área nos diversos tratamen tos de densidade.

Com relação aos cravos com cälice rachado, por planta, foi encontrada diferença entre a maior e a menor densidade (Tabela 4 e Figura 2). O aumento do nümero de cravos rachados por planta, com o decréscimo da densidade de plantio também foi encontrado por FAIRFIELD (1965).

Segundo CONNORS (1916), a tendência de rachamento do cälice é hereditäria, mas existe uma grande influéncia das condições ambientais na frequência do aparecimento desse defei to. Pode-se sugerir que a maior ocorrência de flores com rachadura de cálice, obtidas no plantio menos denso seja consequéncia do aumento no número de pétalas nessa densidade.

Apesar da diminuição no número de cravos rachados por planta, com o aumento da densidade de plantio, as produções de cravos com cálice rachado por hectare não foram diferentes entre si (Tabela 3). Esses resultados foram semelhantes aos obtidos por MENHENETT et alii (1967) e contrariando aos de SEAGER (1969), para quem o aumento da densidade promoveu um maior nümero de cravos rachados por unidade de área. 0 acréscimo no número de plantas da menor para a maior densidade de plantio foi suficiente para equilibrar a diminuição do número de cravos rachados por planta, sem afetar a produção por hectare.

Não foram encontradas diferenças entre os comprimentos das hastes florais nas diferentes densidades de plantio experimentadas, confirmando os resultados obtidos por HOLLEY $\varepsilon$ LEHMAN (1961) e SEAGER (1969). Entretanto, o peso da haste 
com flor foi diferente para as densidades de plantio estudadas, contrariando os resultados obtidos por HOLLEY \& LEHMAN (1961) e SEAGER (1969).

Os pesos das hastes com flor para as maiores densidades foram inferiores aos obtidos na menor densidade (Figura 3).

o diâmetro dos cravos para a maior densidade foi diferente do obtido na menor densidade. O diâmetro aumentou com - decréscimo da densidade, não confirmando os resultados obti dos por HOLLEY \& LEHMAN (1961) e sugerindo que na menor densi dade, as plantas apresentaram menor competição entre si pois tinham maior espaço, consequentemente maior quantidade de nutrientes, àgua e luz e, por isso maior peso de haste floral e maior diâmetro da corola. Segundo JANICK (1966), a diminuição dos órgãos vegetais em tamanho, número, ou em ambos, ocorre como uma reação à pressão de população, após iniciada a competição entre as plantas. Em outros paises, a padronização de cravos visando a comercialização considera o diāmetro da flor como parâmetro de qualidade (HOLLEY \& LEHMAN, 1961), enquanto que no Brasil essa característica não é avaliada.

CONCLUSÕES

a) A produção de cravos com cālice inteiro por hectare aumentou com a densidade porēm, não houve influência da densi dade sobre a produção de cravos com cālice inteiro por plan= ta;

b) a densidade não influenciou a produção de cravos com cálice rachado por hectare, mas a produção de cravos com cālice rachado por planta aumentou com o decréscimo da densidade de plantio;

c) a densidade de plantio não influenciou o comprimento da haste floral de cravos;

d) o peso da haste floral e o diâmetro da corola dos crạ vos aumentam com o decréscimo da densidade de plantio. 


\section{SUMMARY}

EFFECTS OF DENSITY OF PLANTING ON QUALITY OF THE FLOWER IN CARNATION CROP

The influence of density of planting on quality of the flower in carnation plants grown invinil houses was studied.

Planting densities of 233,333,176,000 and 116,667 plants per hectare were obtained by using planting spacings of $0.20 \mathrm{~m}$ between rows and $0.15 \mathrm{~m}, 0.20 \mathrm{~m}$ and $0.30 \mathrm{~m}$ between plants in the rows. Data on number of flowers with perfect calyx and on number of flowers with splitted calyx both per plant and per hectare were taken, as well as on flower plus flower stalk weight and on length and diameter of corola.

As far as planting densities are concerned, there was an increase in production of flowerswith perfect calyx per hectare and a decrease in production of flowers having splitted calyx per plant, flower plus flower stalk weight and corolla diameter as planting density increases. There was no influence of the planting density on the production of perfect calyx flower per plant, splitted calyx flower production per hectare and flower plus flower stalk length.

\section{LITERATURA CITADA}

BUNT, A.C.; SHEARD, G.F., 1967. Observations on the effect of time of planting and plant density on the yield and flower quality of the carnation (Dianthus caryophyzzus). Journal Horticultural Science 42: 263-275.

CONNORS, C.H., 1916. Factors causing the spliting of carnation calyces. Report, New Jersey Agricultural, Experimental Station Paterson, p. 83-89.

DURKIN, D.J.; JANICK, J., 1966. The effect of plant density on greenhouse carnation production. Proc. American Society Horticultural Science 89: 609-614. 
FAIRFIELD, 1965. Carnation experiments. In: 6th Report. Experimental Horticultural Station, Fairfield, p. 75-79.

GARCIA, J.A.; AZURMENDI, J.M.0., 1971. Cultivo intensivo del clavel. Hojas Divulgadoras n. 22/23, 32p.

HOLLEY, W.D.; LEHMAN, F.M., 1961. Spacing patterns for carnation. Florists Exchange 136: 62-64.

JANICK, J., 1966. A Ciência da Horticultura, Rio de Janeiro, USAID, $485 \mathrm{p}$.

JENSEN, H.E.K., 1973. Planteafstande og knibningers indflyde! se pa stilkudbytte, kvalitet og okonomi hos nelliker, (Dianthus caryophyzlus, L.). Tidsskrift for Planiteavl 77: $337-351$.

KINNINGS, J., 1965. Experiments with carnations at Fairfield, Progress Repor. Experimental Husbandry Farms and Horticulture Stations, London: 47-49.

KIPLINGER, D.C., 1963. Spacing and nutrition tests on carna tions. Ohio Florists Association Bulletin, Columbus, ne $405: 6$.

KOON, G., 1962. Modern carnation production. Chio Florists' Association Bulletin, Columbus, n? 392: 1-8.

LAURIE, A.; RIES, V.H., 1950. Floriculture; fundamentals and practices. 2a ed., New York, McGraw-Hill, 525 p.

LOCKIE, G.D.; BUTTERS, R.S., 1956. Further cultural developmen ts in glasshouse crops at Fernhurst Carnations. Fernhurst Bulletin, Fernhurst, 3: 20-23.

LOCKIE, G.D., 1961. Spacing arrangements for carnation produc tion. Britsh Nation Carnation Society hearbook, 1960-61, London: 29-33.

MENHENETT, R.; LOCKIE, G.D.; LOWCOCK, A.M., 1967. Effects of plant density and flower stem cutting length on the perpetual flowering carnation. Experimental Horticulture, London, 17: $9-14$. 
MIRANDA, M.A. de L., 1964. Cravo. In: São Paulo, Secretaria da Agricultura. Padronização de produtos horticolas, São Paulo, $56 \mathrm{p}$.

PIMENTEL GOMES, F., 1976. Curso de estatística experimental, 6a ed., Piracicaba, Graf. Benetti, 430 p.

SEAGER, J.C.R., 1969. Effect of spacing and stopping on flower production in the perpetual flowering carnation. Irish Journal Agricultural, Research 8: 261-270.

SHEARD, G.F.; BUNT, A.C., 1963. The effect of planting date and spacing on the yield of the carnation, with special reference to the economic aspects. Report of the Glasshouse Crops Research Institute, 1962, Littlehampton: 133-137.

SHEARD, G.F., 1967. Spacing, stopping and timing. Bulletin. Ministry of Agriculture, London, 151: 145-146.

SZENDEL, A.J., 1937. Calyx splitting of carnation flower: preliminary report on nutrition experiments. Proc. American Society Horticultural Science 35: 781-787.

VONK-NOORDEGRAAFF, C., 1969. Wanneer gaan we anjers planten? hoeveel planten per $\mathrm{m}^{2}$ ? Vakblad voor de Bloemisterij 24: 277.

WELKE PLANTHOEVEELHEDEN zijn bij anjers verantwoord?, 1968. Vakblad voor de Bloemisterij 23: 671. 
